\title{
Assessment of Knowledge and Attitude towards Ergonomics in Dental Practice
}

\section{Shenoi R ${ }^{1}$, Jain $S^{2 *}$, Kolte $V^{3}$, Shenoi $\mathrm{P}^{4}$ and Kartik DS ${ }^{5}$}

${ }^{1}$ Professor and Head of the Department of Oral and Maxillofacial Surgery VSPM Dental College and Research Centre, India

${ }^{2}$ Junior Resident, Department of Oral and Maxillofacial Surgery VSPM Dental College and Research Centre, India

${ }^{3}$ Professor, Department of Oral and Maxillofacial Surgery VSPM Dental College and Research Centre, India

${ }^{4}$ Professor and Head of the Department of Conservative dentistry and Endodontics VSPM Dental College and Research Centre, India

${ }^{5}$ Assistant Professor, Department of Musculoskeletal Science, VSPM'S College of Physiotherapy, India

*Corresponding author: Dr. Shruti Jain, Junior Resident, Department of Oral and Maxillofacial Surgery VSPM Dental College and Research Centre, Nagpur, India, Tel: +917066625759; Email: drshroo.jain@gmail.com

\section{Abstract}

Objective: To analyze prevalence of knowledge in dentists regarding ergonomics in dentistry.

Background: Ergonomics has become the overseer of all the professions in past decade. An inside out of ergonomics could definitely aid in reducing the number of dentists retiring due to musculoskeletal disorder.

Method: A self-administered, close ended questionnaire consisting of 10 questions regarding ergonomics was distributed among the postgraduates and dental practitioners to assess the knowledge about basic ergonomics in dentistry.

Results: Overall it was observed that the prevalence of knowledge regarding ergonomics is scarce among dentists whereby only $25.6 \%$ respondents have attended any workshop or lecture for ergonomics. The awareness pertaining to four handed and six handed dentistry was higher vis a vis other ergonomical aspects of dentistry. The rest such as neutral position, microbreaks, stretch breaks etc saw lesser awareness.

Conclusion: The low prevalence of knowledge of ergonomics is an alarming situation. The need of the hour is instilling appropriate and elaborate knowledge among the dentists of ergonomics thereby lowering the associated musculoskeletal disorders.

Application: Present study has thrown light on poor awareness of ergonomics which calls for organizing more workshops and lectures for the same thereby promoting an ergonomically sound practice of dentistry.

Keywords: Musculoskeletal Disorder; Neutral Position; Micro Breaks; Stretch Breaks; Four Hande Dentistry

\section{Practioner Summary}

To analyze prevalence of knowledge in dentists regarding ergonomics in dentistry. A self-administered, close ended questionnaire regarding ergonomics was distributed among the postgraduates and dental practitioners to assess the same. This paper throws light on the awareness of the dentists about ergonomics thus being able to conclude 
that the need of the hour is not just to promote ergonomic practice but first instill the knowledge regarding the same. This paper also reviews various ergonomically sound ways of practice.

\section{Introduction}

The realm of Ergonomics affects all of us but the knowledge related to it prevails erratically amongst us. It is an understanding of getting beyond the confinements of workplace so as to optimize the working conditions for the tall as well as short or the fat as well as the thin and so on [1]. Past decade has seen ergonomics emerging as an overseer of almost all the practiced professions where dentistry is no different.

Occupational hazard in dentistry requires addressing musculoskeletal disorders majorly. In a study conducted on 200 practicing dentists in the Delhi - NCR area, $64.5 \%$ of the clinicians have experienced symptoms of MSDs [2].

Rolander and Bellner, [3] who surveyed Swedish dentists in 2001, showed that $86 \%$ of them suffering from MSDs. AlWazzan, et al. [4] found that $54 \%$ of 204 dental professionals experienced neck pain in a region of Saudi Arabia.

These high percentage of dentists experiencing MSDs are harmonious with other studies conducted in various parts of the world by Leggat, et al. [5] in Australia, Sartorio, et al. [6] in Italy, Lalumandier, et al. [7] in USA and many others. Surveys also show that most common reason of dentists retiring early is MSDs [8].
Occasional backache or neck ache is not a cause for alarm, if regularly occurring pain or discomfort is ignored, the cumulative physiological damage can lead to an injury or a career-ending disability [9]. So, the need of the hour is ergonomical prevention in those not suffering from regular discomfort and ergonomical intervention in those experiencing initial symptoms whereas established MSDs would require a medical therapy. But to practice an ergonomically sound dentistry its first important to be aware of it. This study aims to assess the awareness of ergonomics in dentistry amongst dental practitioners in the central region of India.

\section{Methodology}

350 practicing dentists in the central India region were sent the questionnaire out of which 281 responded. The questionnaire was distributed both in hardcopy and via Google forms to the clinicians on their habits and knowledge about ergonomics. The given questionnaire consisted of 10 close ended questions.

\section{Results}

A total of 281 participants filled the questionnaire out of which 225 were practicing dentists and remaining were post graduates. In the present study out of 281 respondents, 184 (65.5\%) were aware of concept of ergonomics in dentistry with 47 of them being unsure about it and 50 (17.8\%) were completely unaware of it. Two hundred and nine out of 281 (74.4\%) never attended a workshop or lecture on ergonomics.

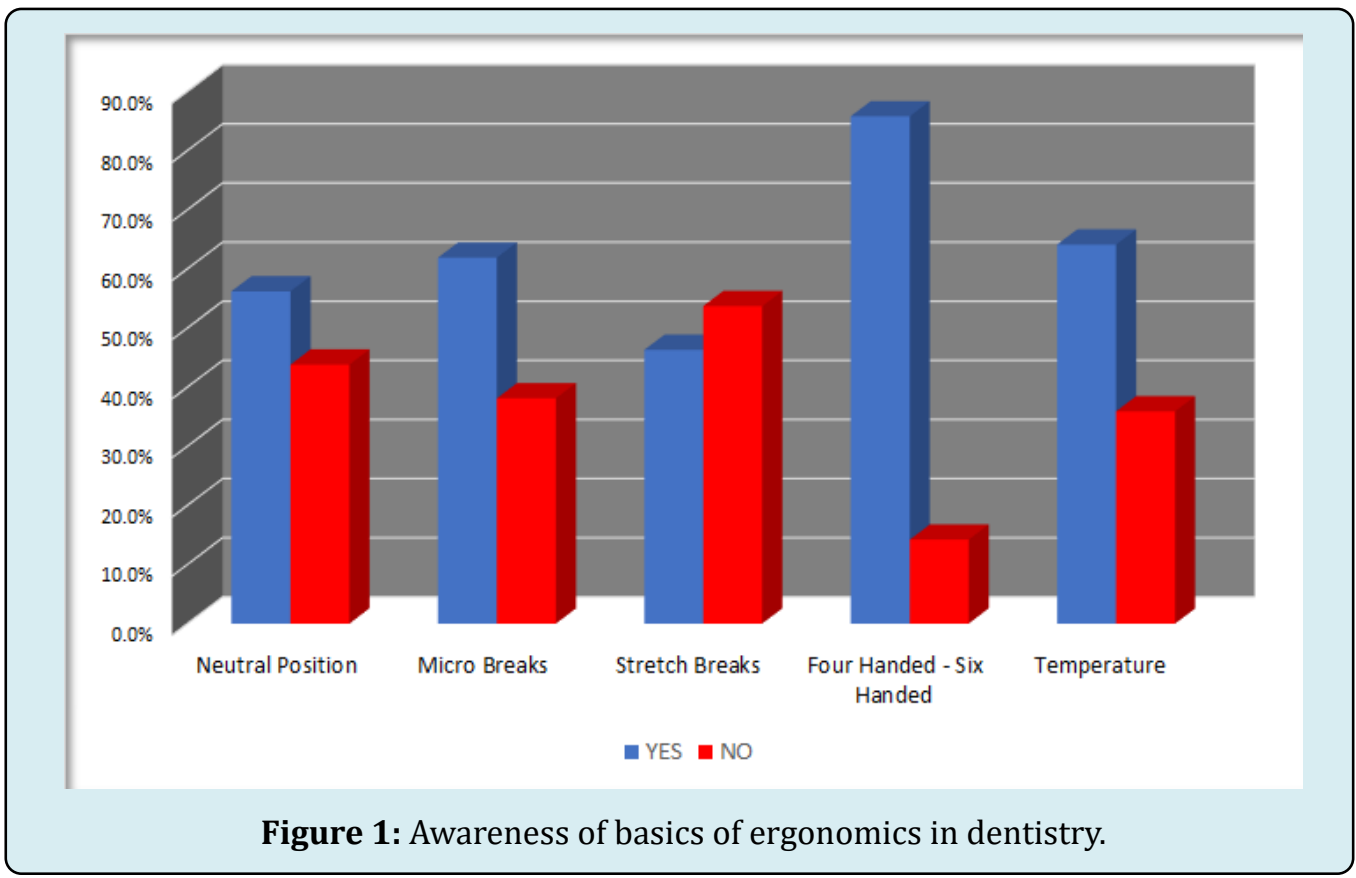


Following this, awareness of basics of ergonomics in dentistry was evaluated through a set of questions whose results are as shown in Figure 1. 56.2\% were aware about the neutral position, $61.9 \%$ were aware about microbreaks, $46.3 \%$ were aware about stretch breaks, $85.8 \%$ were aware about four handed and six handed dentistry, 64.1\% were acquainted with effect of temperature on ergonomic.

After the evaluation of knowledge a glimpse of implementation of these modalities were assessed by asking so as to which feature the practice maximum in their day to day clinic. The results were as per the pie diagram figure 2 .

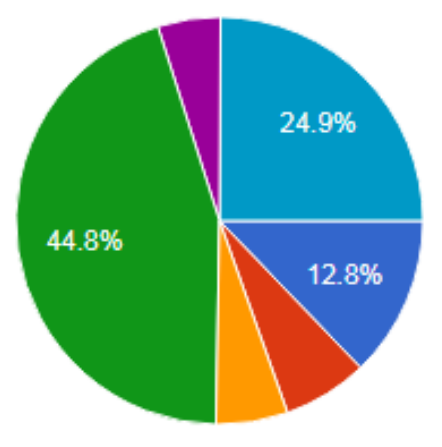

Neutral position

Microbreaks

Stretch breaks

Four handed or six handed dentistry

Ergonomically designed new instruments

No, I have practiced none of the above

Figure 2: Pie diagram of awareness of basics of ergonomics in dentistry.

When asked about whether the principle of ergonomics applies only to the position of dentist and not the patient, 52.3\% answered a "no", 20.3\% answered "yes" whereas $27.4 \%$ of them were unaware.

Furthermore, when asked about the awareness regarding newly available products in the market that are specifically designed on the principles of ergonomics only $34.9 \%$ of them were aware of it.

\section{Discussion}

Pain experienced by dentists is no more a new agenda but preventing its occurrence is now in lime light. Repetitive motions, awkward postures for reaching the oral cavity and static postures are all inevitable in dentistry but ergonomics can help combat these risk factors of MSDs [10,11].

In our survey $66.5 \%$ of dentists were aware of ergonomics existing in dentistry but only handfuls of them have ever attended a workshop or a lecture on the same. In our study $25.6 \%$ attended seminar or a workshop related to ergonomics which was not harmonious with only $7 \%$ in a study conducted by Khan, et al. [11] in Malaysia.

\section{Neutral Position}

In our study $56.2 \%$ percentage of dentists were aware of neutral position. In order to assess the confines of oral cavity, bending and twisting of dentists is not unusual. For most of the time these postures are beyond the acceptable range of flexion, extension, adduction or abduction of a joint. It is very important to clearly understand these neutral positions for an effective practicing of the same.

Whenever the head is bend the muscles of neck contract to keep head from falling thereby causing fatigue of neck muscles if bending is for prolonged duration. Though a straight neck posture is barely possible for a dentist, a flexion till 20-25 degrees is permissible without causing much damage $[12,13]$.

Shoulder slouching is again not uncommon in dentists that needs to be consciously corrected while working by knowing the ill effects of it. Whenever the shoulder is bending forward it leads to increase in interscapular distance thereby causing stretching of related muscles [13].

The arms and the forearms need to be kept as close to body as possible. The angle between arm and forearm ideally should be 90 degrees but a flexion till 60 degrees and extension till 100 degrees is acceptable. At all times forearms should be supported which is best facilitated by the chairs that have fully adjustable hand rest.

Hand should be in line with forearm with barely permissible adduction abduction extension and flexion. Hand could be bent on the side of little finger and to be avoided towards the thumb. Spinal curve should be maintained by 
an apt back rest especially at the lumbar region. Hip to thigh angle should be approximately 110 degrees which implies that hip should be slightly higher than knees. Such a posture also aids in positioning closer to patient. The knee end of thigh should not be resting on the seat so as to prevent the leg from becoming numb. Both the feet should be in contact with floor entirely with all sorts of foot controls in near reach [13].

\section{Microbreaks}

In our study $61.9 \%$ dentists knew about microbreaks but only $6.9 \%$ practiced it which is incontrast with a study by Kierklo, et al. [14] 32.73\% of dentists work without any break, $36.37 \%$ take one break a day, $16.36 \%$ have 2 breaks a day, and $8.18 \%$ rest after each patient $[14,15]$.

This discrepancy in the awareness and its implementation probably lies because of lack of thorough knowledge and its consequences. There are two key points to practicing microbreaks- i) duration ii) frequency. Too long breaks might result in inability to work whereas too short breaks or breaks placed too far apart might not heal the tissues at the rate in which they are damaged [16-18].

\section{Stretch Breaks}

Having operators take frequent breaks and reverse their positions is integral in an effective injury prevention program. Directional stretches such as rotation, side-bending or extension can he performed either while sitting on chair or outside the operating room. Directional stretching involves a component that generally is in the opposite direction of that in which the operator frequently works thereby addresses the muscle imbalances that tend to develop. Frequent stretching breaks address the detrimental physiological changes that can develop while working in optimal or awkward prolonged static postures [8].

These breaks have proven to drastically stop the progress of musculoskeletal disorders but our study shows a bare awareness of it and almost none practicing it.

\section{Operator and Patient Position}

A common mistake among dentists is positioning patients too high. This causes elevation of the shoulders and abduction of the arms, leading to prolonged static muscular tension in the neck and shoulders. Operators should take the time to position their patients properly for mandibular and maxillary procedures. Supine position of the patient increases accessibility of both the sides with less bending and twisting of operator.

For the operator it is necessary to keep shifting the load from one group of muscles to another while working by

- Changing positions between the procedure

- Alternating between standing and sitting

- $\quad$ Scheduling i.e. not appointing a similar treatment back to back and alternating a log and short procedure with reduced appointments on the day of a difficult procedure.

\section{Four Handed Six Handed Dentistry}

This is a chair side ergonomic arrangement about which maximum respondents were aware (85.8\%) which was consistent with the percentage of dentists practicing it (44.8\%). A definite position of the dentist and the trained assistant is designed so as to avoid twisting and bending to grab an instrument as well as for the procedure. An irony of this arrangement is the consequent static posture that the dentist acquires. This can be overcome by a conscious change in position during the procedure $[9,10]$.

\section{Conclusion}

By now it is well established that musculoskeletal disorders are common amongst the dentist due to awkward postures. The call is for an ergonomic intervention for increased productivity and delayed retirement of dentists. This intervention is not medicinal rather it's a practice at personal level which can only come by a thorough knowledge of the topic which was found to prevail only fairly.

\section{Key Points}

- Musculoskeletal disorders are the most common reason for early retirement of dentists.

- All the joints should be kept close to their neutral position while working.

- Micro breaks should be of apt duration and in frequent intervals

- Four handed or six handed dentistry should be performed with a caution of not being seated in a static posture for too long.

\section{References}

1. Gunning J, Eaton J, Ferrier S, Frumin E, Kerr M, etal. (2001) Ergonomic handbook for the clothing industry. Union of Needletrades, Industrial and Textile Employees, Toronto.

2. Batra H, Rajvanshi H, Anshul K, Singh I, Chhabra N, et al. (2015) An Estimation of Awareness among Practicing Dentists about Proper Ergonomic Practice and its Implications in Delhi-National Capital Region. International Journal of Scientific Study 3(7): 70-75.

3. Rolander B, Bellner AL (2001) Experience of musculo- 
skeletal disorders, intensity of pain, and general conditions in work--The case of employees in non-private dental clinics in a county in southern Sweden. Work 17(1): 65-73.

4. Al Wazzan KA, Almas K, Al Shethri SE, Al-Qahtani MQ (2001) Back \& neck problems among dentists and dental auxiliaries. J Contemp Dent Pract 2(3): 17-30.

5. Leggat PA, Smith DR (2006) Musculoskeletal disorders self-reported by dentists in Queensland, Australia. Australian dental journal 51(4): 324-327.

6. Sartorio F, Vercelli S, Ferriero G, D’Angelo F, Migliario $M$, et al. (2005) Work-related musculoskeletal diseases in dental professionals. 1. Prevalence and risk factors. Giornale italiano di medicina del lavoro ed ergonomia 27(2): 165-169.

7. Lalumandier JA, McPhee SD, Parrott CB, Vendemia M (2001) Musculoskeletal pain: prevalence, prevention, and differences among dental office personnel. General dentistry 49(2): 160-166.

8. Gupta A, Bhat M, Mohammed T, Bansal N, Gupta G (2014) Ergonomics in dentistry. International journal of clinical pediatric dentistry $7(1)$ : 30-34.

9. Valachi B, Valachi K (2003) Mechanisms leading to musculoskeletal disorders in dentistry. The Journal of the American Dental Association 134(10): 1344-1350.

10. Valachi B, Valachi K (2003) Preventing musculoskeletal disorders in clinical dentistry: strategies to address the mechanisms leading to musculoskeletal disorders. The Journal of the American Dental Association 134(12):
1604-1612.

11. Khan SA, Chew KY (2013) Effect of working characteristics and taught ergonomics on the prevalence of musculoskeletal disorders amongst dental students. BMC musculoskeletal disorders 14(1): 118.

12. Rodgers SH (1986) Ergonomics Design for People at Work, The Design of Jobs, Including Work Patterns, Hours of Work, Manual Materials Handling Tasks, Methods to Evaluate Job Demands and the Physiological Basis of Work by Ergonomics Group Eastman Kodak Company.

13. Shaik AR (2015) Dental ergonomics: Basic steps to enhance work efficiency. Archives of Medicine and Health Sciences 3(1): 138-144.

14. Kierklo A, Kobus A, Jaworska M, Botuliński B, Pawińska M (2008) Rest breaks as an element of the dentists' health hazards prevention. The analysis in Podlasie province. Implantoprot Stom Klin 9(3): 39-42.

15. Tucker P, Folkard S, Macdonald I (2003) Rest breaks and accident risk. The Lancet 361(9358): 680.

16. Oberg T, Karsznia A, Sandsjo L, Kadefors R (1995) Work load, fatigue, and pause patterns in clinical dental hygiene. Journal of dental hygiene 69(5): 223-229.

17. Hille G, Seget A, Pytko-Polonczyk J (2015) Ergonomics in the professional work of the dentist-a questionnaire survey. In Dental forum 41(2): 45-52.

18. Akesson I, Hansson GA, Balogh I, Moritz U, Skerfving S (1997) Quantifying work load in neck, shoulders and wrists in female dentists. International archives of occupational and environmental health 69(6): 461-474. 